\title{
Role of matrix metalloproteinases in the pathogenesis of intracranial aneurysms
}

\author{
Xiaoran Zhang, MD, ${ }^{1}$ William J. Ares, MD, ${ }^{1}$ Philipp Taussky, MD, ${ }^{2}$ Andrew F. Ducruet, MD, ${ }^{3}$ and \\ Ramesh Grandhi, MD²
}

\begin{abstract}
${ }^{1}$ Department of Neurological Surgery, University of Pittsburgh Medical Center, Pittsburgh, Pennsylvania; ${ }^{2}$ Department of Neurosurgery, University of Utah School of Medicine, Salt Lake City, Utah; and ${ }^{3}$ Department of Neurological Surgery, Barrow Neurological Institute, Phoenix, Arizona
\end{abstract}

\begin{abstract}
Intracranial aneurysms (IAs) are a result of complex interactions between biochemical and mechanical forces and can lead to significant morbidity if they rupture and cause subarachnoid hemorrhage. This review explores the role of matrix metalloproteinases (MMPs) in the pathogenesis and progression of IAs. In addition to providing a review of the normal function of MMPs, it is intended to explore the interaction between inflammation and abnormal blood flow and the resultant pathological vascular remodeling processes seen in the development and rupture of IAs. Also reviewed is the potential for the use of MMPs as a diagnostic tool for assessment of aneurysm development and progression.
\end{abstract}

https://thejns.org/doi/abs/10.3171/2019.4.FOCUS19214

KEYWORDS matrix metalloproteinase; intracranial aneurysm; extracellular matrix; vascular remodeling; inflammation

$\mathrm{I}$ NTRACRANIAL aneurysms (IAs) are characterized by localized structural changes to the arterial vessel wall that lead to weakened wall integrity and vessel dilation at the point of weakness. IAs are often found near vessel bifurcations, probably as a result of turbulent flow at those areas. Unruptured IAs have a prevalence of $1 \%-5 \%$ in the population older than 30 years of age. ${ }^{53,56}$ Known risk factors for IA formation and rupture include cigarette smoking, hypertension, and familial history. ${ }^{56}$ IA rupture results in subarachnoid hemorrhage (SAH), a condition with high rates of morbidity and mortality. In patients with $\mathrm{SAH}$ secondary to rupture of an IA, $12 \%$ die prior to receiving medical attention, and of those who are admitted to the hospital, $40 \%$ die within the first month. ${ }^{29,57}$ One-third of the individuals who survive SAH have significant permanent neurological deficits. ${ }^{56}$

Histopathologically, IAs exhibit loss of the internal elastic lamina (IEL), atrophy of the media, and abnormal patterns of extracellular matrix (ECM) expression. ${ }^{9}$ Although the mechanisms underlying these histopathological changes are poorly understood, there is growing evidence that inflammation plays an important role in the disease process. ${ }^{13}$ Matrix metalloproteinases (MMPs) are molecules of particular interest, not only because they are upregulated in inflammatory states but also because of their intrinsic ability to degrade ECM components and their role as the main effector molecules in ECM remodeling. Numerous studies have demonstrated that MMP-2 and MMP-9 play a significant role in aneurysm formation and progression., 5,36 As gelatinases, MMP-2 and MMP-9 are responsible for the degradation of major components of the vascular ECM and IEL, including elastin and collagen types I and III.

Despite the significant public health implications of IAs, preventive management of these lesions has been hindered by the limited understanding of the mechanisms of their formation, growth, and rupture. Further elucidating the role that MMPs play in the pathogenesis of IAs may help facilitate the development of screening tools for early intervention, as well as potentially stratifying IAs according to risk of rupture.

\section{MMP Biology}

First discovered in 1962 as a collagenase that mediated the resorption of the tadpole tail, MMPs are a family of structurally related proteins that mediate a wide variety of biological processes through enzymatic activity. ${ }^{23}$ MMPs

ABBREVIATIONS AAA = abdominal aortic aneurysm; ECM = extracellular matrix; IA = intracranial aneurysm; IEL = internal elastic lamina; IL = interleukin; MMP = matrix metalloproteinase; $\mathrm{SAH}=$ subarachnoid hemorrhage; TIMP = tissue inhibitor of metalloproteinases; $\mathrm{TNF} \alpha=$ tumor necrosis factor $-\alpha$; VSMC = vascular smooth muscle cell; WSS = wall shear stress.

SUBMITTED March 1, 2019. ACCEPTED April 9, 2019

INCLUDE WHEN CITING DOI: 10.3171/2019.4.FOCUS19214. 
play a significant role in eliciting changes in the microarchitecture of tissues and also contribute to the development of cell lines. These functions include initiating structural changes to proteins within the ECM and affecting chemotaxis, cell survival, and proliferation. MMPs are classified into 6 groups based on their functionality: collagenases, gelatinases, stromelysins, matrilysins, membrane-type MMPs, and others..$^{62,63}$ The majority of the proteins found in the ECM are known to be substrates of MMPs. Specifically, MMP-9 has been demonstrated to degrade elastin, and MMP-2 has been shown to degrade collagen types I and III. ${ }^{1,38}$ In addition, MMPs and their cleaved products can modify the downstream effects of cell-signaling molecules such as growth factors, cell adhesion molecules, cytokines, and other MMPs. Although functionally different, all MMPs share a high degree of structural homology that includes a propeptide, a catalytic domain, a hinge region, and a hemopexin domain (Fig. 1).

MMPs are synthesized and secreted into the bloodstream as zymogens (pro-MMPs) by vascular smooth muscle cells (VSMCs), macrophages, and fibroblasts. . $^{15,20,21}$ The conversion from pro-MMPs to enzymatically active MMPs requires the proteolytic cleavage of the propeptide.$^{60}$ Levels and activities of proteinases, including other MMPs and MMP inhibitors, tightly regulate this process. ${ }^{12,62} \mathrm{~A}$ variety of factors also regulate the expression of MMPs, including inflammatory cytokines such as tumor necrosis factor- $\alpha(\mathrm{TNF} \alpha)$ and interleukin-1 $\beta$ (IL-1 $\beta)$; vascular factors such as endothelin A; ECM proteins; and mechanical forces such as stretch and shear stress. $3,6,32,33,49$ The main endogenous inhibitors of MMP activity are tissue inhibitors of metalloproteinases (TIMPs), which function by binding the C-terminal domain of MMPs. ${ }^{62,63}$ Thus, the complex interplay between inducers of $M M P$ expression and activation and TIMPs has a key role in the development and pathogenesis of IAs.

\section{Vascular Remodeling in IAs}

The development of cerebral aneurysms can be divided into 3 stages: initiation, growth, and rupture ${ }^{56}$ Although the pathophysiology behind each stage of aneurysm development has not been clearly delineated, abnormal blood flow and inflammation have been recognized to play key roles in IA pathogenesis. ${ }^{14,22}$ The initiation phase of aneurysm development is thought to be due to the effect of abnormal blood flow on a blood vessel that is already predisposed to aneurysm formation..$^{56}$ This predisposition is known to be influenced by a combination of genetic, physiological, and environmental factors..$^{35}$ It is well known that individuals with genetic defects of collagen production (e.g., Ehlers-Danlos syndrome type IV) or those who smoke cigarettes are at increased risk for the development of aneurysms. ${ }^{40,52}$ Sites of aneurysm formation are generally localized to areas of vessel bifurcation curvatures, and aneurysms are thought to be due to intimal injury secondary to altered laminar blood flow in these areas. ${ }^{22}$ Blood flow exerts pressure and wall shear stress (WSS) on endothelial cells lining vessel lumens. In studies of human and animal subjects, WSS has been
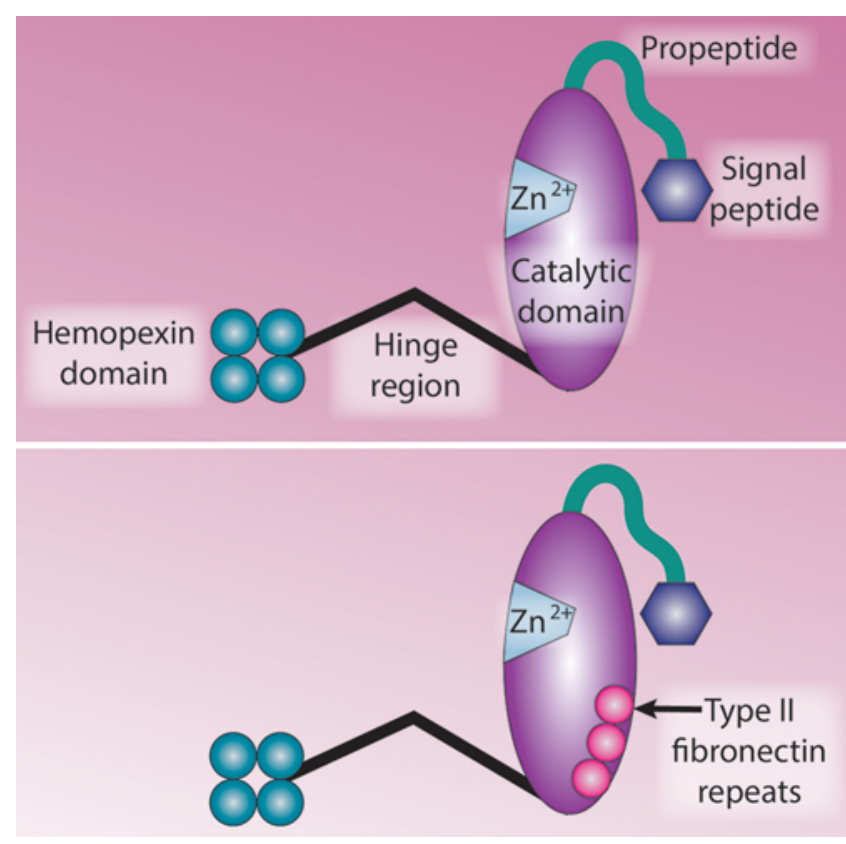

FIG. 1. MMP domain structures. All MMPs share a high degree of structural homology that includes a propeptide, a catalytic domain, a hinge region, and a hemopexin domain. Upper: Collagenases, stromelysins, and other MMPs. Lower: Gelatinases (MMP-2, MMP-9). Copyright Department of Neurosurgery, University of Utah. Published with permission.

found to be significantly greater at vessel bifurcations..$^{11,59}$ Changes in WSS can be detected by mechanically sensitive endothelial cells, leading to the initiation of the vascular remodeling process. ${ }^{59}$

A hallmark of vascular remodeling in IAs is atrophy of the tunica media-a layer of smooth muscle responsible for providing structural support to the arterial wall-leading to localized weakening of the arterial wall and the possibility of vessel dilation. This phenomenon is believed to be mediated by the apoptosis and migration of the primary cellular component of the tunica media, VSMCs..$^{24,42,55}$ Kondo and colleagues ${ }^{41}$ elucidated the process of atrophy of the tunica media during aneurysm formation by ligating the common carotid arteries of rats and inducing prolonged hypertension over several months. Histopathological analysis of the vessel walls demonstrated that shortly after the disappearance of the IEL, an early marker of vascular remodeling prior to vessel dilation, VSMC density remained the same between control and experimental groups. Corresponding with progressive vessel wall dilation, the VSMCs became more disorganized and displayed decreased intracellular volume and a reduction in cellular density. In the mature aneurysm, VSMC density was vastly reduced and the remaining cells were poorly organized with irregular cellular morphology. By using terminal deoxynucleotidyl transferase dUTP nickend labeling (TUNEL) staining and light microscopy, the authors further showed that the VSMCs demonstrated the cardinal signs of apoptosis: cell shrinkage, chromatin condensation, and formation of apoptotic bodies within the cells. ${ }^{55}$ Although compelling, the above results have not 
Lumen

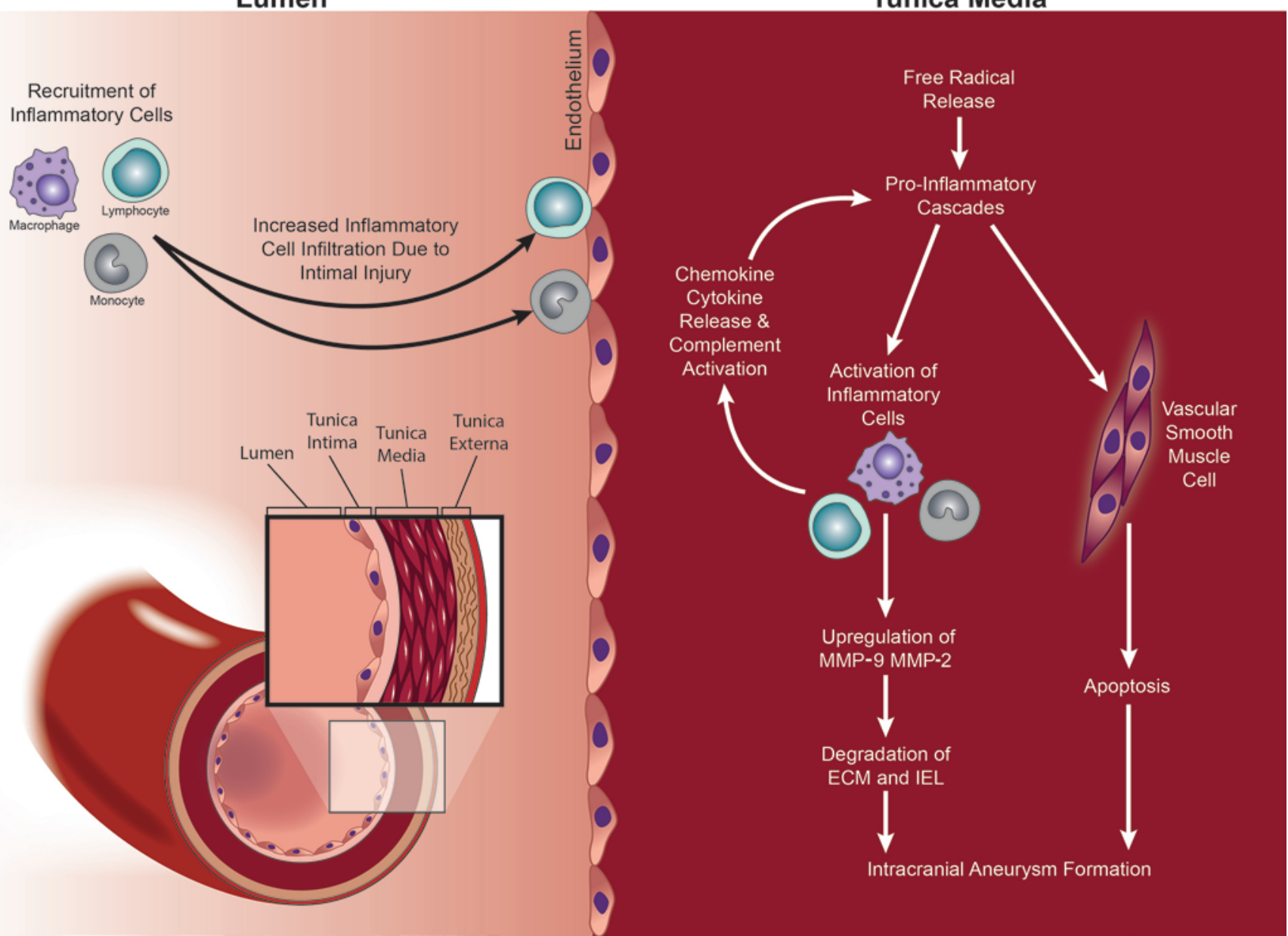

FIG. 2. MMPs mediate IA formation via a process of vascular remodeling. Intimal injury and increased WSS leads to recruitment and infiltration of inflammatory cells into the vessel wall. Release of free radicals, proinflammatory chemokines, and cytokines, and activation of the complement cascade mediate upregulation of MMP-2 and MMP-9. The consequent proteolytic process results in the degradation of the ECM within the tunica media and altered structure of the vessel wall with IA formation. Copyright Department of Neurosurgery, University of Utah. Published with permission.

been replicated in human studies. Large studies of human aneurysm wall samples demonstrate variable VSMC density among different aneurysms and even within the same aneurysm. ${ }^{17,37,43}$ In some samples there is a higher density of VSMCs in the aneurysm wall than in the parent vessel. ${ }^{16,17}$

The causes of apoptosis and remodeling of VSMCs are multifactorial; however, oxidative stress in the vessel wall also clearly plays a significant role. ${ }^{43,61}$ Mechanical stress has been shown to increase production of free radicals in the affected vessel walls. ${ }^{30}$ Free-radical formation in cerebral arteries is driven by nicotinamide adenine dinucleotide phosphate oxidase (NADPH) and cyclooxygenases. Although these substrates are functionally active in healthy endothelial cells, the increased production of free radicals (superoxide and hydrogen peroxide) can lead to activation of inflammatory cascades, resulting in increased migration of MMP-releasing monocytes and macrophages and the activation of pro-MMPs. Increased regional MMP activity ultimately contributes to vessel wall remodeling and weakening and to eventual aneurysmal dilation (Fig. 2). ${ }^{61}$

\section{MMPs and Aneurysm Development and Rupture \\ Role of MMPs in Abdominal Aortic Aneurysm Pathogenesis}

Several studies have linked MMP expression to aneurysm development and progression in the setting of abdominal aortic aneurysms (AAAs). At a cellular level, Ishii and Asuwa ${ }^{31}$ reported increased MMP-2 and MMP-9 immunohistochemical staining at the sites of intimal tears in patients with acute aortic dissection. Observational studies have further supported this association between elevated $M M P$ expression at the tissue level and the presence of progressive AAA.$^{50,65}$ Furthermore, gene knockout models have confirmed the critical role of MMPs in the development of AAAs-MMP-9 knockout models were unable to develop AAAs despite the presence of a chemi- 
cal stimulus. Interestingly, transfection of the $M M P-9$ gene restored the ability to express MMP-9 and the subsequent development of AAAs. ${ }^{45}$

Circulating levels of MMPs have been shown to be elevated in patients with AAA; plasma levels of MMP-9 are higher in patients with AAA than in normal or athero-occlusive controls..$^{27,47}$ In patients with smaller AAAs (3-5 cm), plasma levels of MMPs were predictive of the expansion of these lesions. ${ }^{44}$ Furthermore, Wilson et al. ${ }^{64}$ demonstrated significantly increased levels of MMP-1 and MMP-9 in patients with ruptured AAAs when compared with individuals with nonruptured AAAs.

\section{Role of MMPs in IA Pathogenesis}

\section{Pathophysiology and Experimental Studies}

Elevated levels of macrophages and lymphocytes found within aneurysm walls support the theory that inflammation plays a key role in IA formation..$^{14,18}$ Chyatte et al. ${ }^{14}$ noted that the number of macrophages assembled on the endothelium and within the adventitia increased with propagation of aneurysmal formation. Infiltration of the vascular wall by macrophages due to damage and freeradical release leads to the recognition of ECM components by macrophage cell surface receptors, stimulating increased production of proinflammatory molecules from macrophages. ${ }^{61}$ Among these molecules are TNF $\alpha$ and IL-8, both of which are potent inducers of MMP-2 and MMP-9 expression and production. ${ }^{48}$ Induction of MMP activity can be triggered by other factors at the site of an aneurysm. Mechanical stretch from abnormal blood flow or hypertension is sensed by endothelial cells, which can produce $\mathrm{TNF} \alpha$, a proinflammatory cytokine and potent stimulator of MMP-2 expression. ${ }^{30,58}$ Mechanical stretch also leads endothelial cells to produce free radicals and nitric oxide, leading to the conversion of pro-MMP-9 to active MMP-9. ${ }^{46,61}$

The central role of MMPs in IA pathogenesis has been demonstrated and validated in numerous experimental studies. Using a rat model of IA, Aoki and colleagues ${ }^{7}$ found that aneurysm growth was closely associated with MMP-2 and MMP-9 expression and that selective inhibition of these MMPs can prevent aneurysm progression. Furthermore, using the same rodent model, Kanematsu and colleagues ${ }^{36}$ demonstrated that aneurysm formation was decreased by systemic depletion of macrophages, which are potent producers of MMP-2 and MMP-9. Conversely, inhibitors of MMP such as TIMP-1 and TIMP-2 have a protective effect against aneurysm initiation and growth. TIMPs inhibit proteolytic activity of MMPs through the formation of protein complexes with their specific partners (i.e., TIMP-1 and MMP-9, TIMP-2 and MMP-2). ${ }^{10}$ In a rat model of cerebral aneurysms, genetic knockout of TIMP-1 or TIMP-2 genes both resulted in increased MMP-2 and MMP-9 enzymatic activity and increased rates of aneurysm formation. ${ }^{8}$ Conversely, in a rat model of AAA, local overexpression of TIMP-1 at the site of the aneurysm prevents its degradation and rupture. ${ }^{4}$ Subsequent studies illustrated that TIMP-1 and TIMP-2 are primarily found in VSMCs and are highly expressed in cerebral aneurysms, suggesting that the apoptosis and migration of VSMCs away from tunica media potentially leads to a shift in MMP/TIMP balance favoring increased MMP enzymatic activities, ECM degradation, and eventually aneurysm progression and possibly rupture. ${ }^{8}$

\section{Clinical Studies}

Evidence for an association between $M M P$ expression and IA predisposition, development, and subsequent rupture has been demonstrated in human clinical studies. Genetic predisposition to increased production of MMPs has been tied to an increased propensity to develop IAs. Peters et al..$^{51}$ demonstrated that a polymorphism in the promoter of MMP-9 was associated with the presence of IA in a small population of people with a familial history of ruptured and unruptured IAs. Additionally, in a casecontrol genetic association study, the presence of a single nucleotide polymorphism (rs243865 C > T) leading to an increased transcription of the $M M P-2$ gene was associated with the presence of ruptured IAs, even after controlling for smoking and hypertension status, thus supporting the role of $M M P$ expression in both the development and rupture of IAs. ${ }^{2}$

Local and systemic MMP activity may also contribute to IA development and, more importantly, rupture. Kim and colleagues ${ }^{39}$ found a localized increase in MMP-9 levels in IA wall samples compared with both the patient's own plasma samples and samples from control patients. Jin et al. ${ }^{34}$ noted higher serum and aneurysm wall levels of MMP-2 and MMP-9 among patients with ruptured IAs compared with those with unruptured aneurysms. These findings corroborated the results of a previous study by Gaetani et al., ${ }^{19}$ which found a difference in the activity of the MMPs elastase and collagenase in the aneurysm walls of patients with aneurysmal SAH compared with those with unruptured aneurysms. In contradistinction, Rojas et al. ${ }^{54}$ found a significantly higher serum level of MMP-9 in patients with any IA compared with controls, but they did not find a difference in levels when comparing patients with ruptured versus unruptured IAs.

Interestingly, medications known to decrease inflammation and block MMP production and activation have been shown to be associated with decreased aneurysm rupture rates. ${ }^{13,26,35}$ In fact, recently published data have suggested that patients taking daily aspirin may have a lower risk of aneurysm rupture. ${ }^{13,26,35}$ Although the exact pathophysiological mechanism remains unknown, current theory relies on aspirin's inhibition of cyclooxygenase-2 (COX-2), which may lead to downstream effects by decreasing the expression of immune modulators that contribute to IA rupture. ${ }^{25}$ Moreover, aspirin has been demonstrated to inhibit the expression of MMP-2 and MMP-9 in macrophages, and it may upregulate the expression of TIMPs. ${ }^{28}$

\section{Future Directions}

Given that differential expression of MMPs has been demonstrated between ruptured and unruptured aneurysmal samples, it stands to reason that MMP concentrations could potentially be used for diagnostic purposes. Quantitative levels of circulating MMPs might potentially serve 
as serum biomarkers for both the presence and rupture status of cerebral aneurysms. Furthermore, falling plasma concentrations of these enzymes might be used as a surrogate for successful endovascular treatment (Weiner $G$, Kattar N, Grandhi R: Matrix metalloproteinases as serum biomarkers for the presence, evolution, and indication of successful endovascular treatment of cerebral aneurysms, presentation at the AANS/CNS Cerebrovascular Section Joint Meeting, Nashville, TN, 2015), as has been shown after endovascular AAA repair. ${ }^{49}$ Future studies involving the assessment of MMP levels within intracranial blood and peripheral blood as well as microRNA and proteomic expression within the walls of aneurysms, both ruptured and unruptured, may help determine whether aneurysm presence, evolution, and rupture are due to a systemic, local, or combined process. Indeed, such studies may lead to the identification of targets for the prevention of aneurysm growth and rupture while also stratifying rupture risk, thus enabling physicians to determine which patients are at highest risk for experiencing a life-threatening hemorrhage.

\section{References}

1. Aimes RT, Quigley JP: Matrix metalloproteinase-2 is an interstitial collagenase. Inhibitor-free enzyme catalyzes the cleavage of collagen fibrils and soluble native type I collagen generating the specific 3/4- and 1/4-length fragments. J Biol Chem 270:5872-5876, 1995

2. Alg VS, Ke X, Grieve J, Bonner S, Walsh DC, Bulters D, et al: Association of functional MMP-2 gene variant with intracranial aneurysms: case-control genetic association study and meta-analysis. Br J Neurosurg 32:255-259, 2018

3. Ali MS, Starke RM, Jabbour PM, Tjoumakaris SI, Gonzalez LF, Rosenwasser RH, et al: TNF- $\alpha$ induces phenotypic modulation in cerebral vascular smooth muscle cells: implications for cerebral aneurysm pathology. J Cereb Blood Flow Metab 33:1564-1573, 2013

4. Allaire E, Forough R, Clowes M, Starcher B, Clowes AW: Local overexpression of TIMP-1 prevents aortic aneurysm degeneration and rupture in a rat model. J Clin Invest 102:1413-1420, 1998

5. Aoki T, Kataoka H, Ishibashi R, Nozaki K, Egashira K, Hashimoto N: Impact of monocyte chemoattractant protein-1 deficiency on cerebral aneurysm formation. Stroke 40:942951, 2009

6. Aoki T, Kataoka H, Ishibashi R, Nozaki K, Morishita R, Hashimoto N: Reduced collagen biosynthesis is the hallmark of cerebral aneurysm: contribution of interleukin-1 $\beta$ and nuclear factor- $\kappa \mathrm{B}$. Arterioscler Thromb Vasc Biol 29:1080-1086, 2009

7. Aoki T, Kataoka H, Morimoto M, Nozaki K, Hashimoto N: Macrophage-derived matrix metalloproteinase- 2 and -9 promote the progression of cerebral aneurysms in rats. Stroke 38:162-169, 2007

8. Aoki T, Kataoka H, Moriwaki T, Nozaki K, Hashimoto N: Role of TIMP-1 and TIMP-2 in the progression of cerebral aneurysms. Stroke 38:2337-2345, 2007

9. Austin G, Fisher S, Dickson D, Anderson D, Richardson S: The significance of the extracellular matrix in intracranial aneurysms. Ann Clin Lab Sci 23:97-105, 1993

10. Brew K, Dinakarpandian D, Nagase H: Tissue inhibitors of metalloproteinases: evolution, structure and function. Biochim Biophys Acta 1477:267-283, 2000

11. Burleson AC, Turitto VT: Identification of quantifiable hemodynamic factors in the assessment of cerebral aneurysm behavior. On behalf of the Subcommittee on Biorheology of the Scientific and Standardization Committee of the ISTH. Thromb Haemost 76:118-123, 1996

12. Cerdà-Costa N, Gomis-Rüth FX: Architecture and function of metallopeptidase catalytic domains. Protein Sci 23:123144,2014

13. Chalouhi N, Ali MS, Jabbour PM, Tjoumakaris SI, Gonzalez LF, Rosenwasser RH, et al: Biology of intracranial aneurysms: role of inflammation. J Cereb Blood Flow Metab 32:1659-1676, 2012

14. Chyatte D, Bruno G, Desai S, Todor DR: Inflammation and intracranial aneurysms. Neurosurgery 45:1137-1147, 1999

15. Fowlkes JL, Enghild JJ, Suzuki K, Nagase H: Matrix metalloproteinases degrade insulin-like growth factor-binding protein-3 in dermal fibroblast cultures. J Biol Chem 269:25742-25746, 1994

16. Frösen J: Smooth muscle cells and the formation, degeneration, and rupture of saccular intracranial aneurysm wall-a review of current pathophysiological knowledge. Transl Stroke Res 5:347-356, 2014

17. Frösen J, Piippo A, Paetau A, Kangasniemi M, Niemelä M, Hernesniemi J, et al: Remodeling of saccular cerebral artery aneurysm wall is associated with rupture: histological analysis of 24 unruptured and 42 ruptured cases. Stroke 35:2287-2293, 2004

18. Frösen J, Tulamo R, Paetau A, Laaksamo E, Korja M, Laakso A, et al: Saccular intracranial aneurysm: pathology and mechanisms. Acta Neuropathol 123:773-786, 2012

19. Gaetani P, Rodriguez y Baena R, Tartara F, Messina AL, Tancioni F, Schiavo R, et al: Metalloproteases and intracranial vascular lesions. Neurol Res 21:385-390, 1999

20. Galis ZS, Muszynski M, Sukhova GK, Simon-Morrissey E, Unemori EN, Lark MW, et al: Cytokine-stimulated human vascular smooth muscle cells synthesize a complement of enzymes required for extracellular matrix digestion. Circ Res 75:181-189, 1994

21. Galis ZS, Sukhova GK, Kranzhöfer R, Clark S, Libby P: Macrophage foam cells from experimental atheroma constitutively produce matrix-degrading proteinases. Proc Natl Acad Sci U S A 92:402-406, 1995

22. Gonzalez CF, Cho YI, Ortega HV, Moret J: Intracranial aneurysms: flow analysis of their origin and progression. AJNR Am J Neuroradiol 13:181-188, 1992

23. Gross J, Lapiere CM: Collagenolytic activity in amphibian tissues: a tissue culture assay. Proc Natl Acad Sci U S A 48:1014-1022, 1962

24. Guo F, Li Z, Song L, Han T, Feng Q, Guo Y, et al: Increased apoptosis and cysteinyl aspartate specific protease-3 gene expression in human intracranial aneurysm. J Clin Neurosci 14:550-555, 2007

25. Hasan D, Hashimoto T, Kung D, Macdonald RL, Winn HR, Heistad D: Upregulation of cyclooxygenase-2 (COX-2) and microsomal prostaglandin E2 synthase-1 (mPGES-1) in wall of ruptured human cerebral aneurysms: preliminary results. Stroke 43:1964-1967, 2012

26. Hasan DM, Mahaney KB, Brown RD Jr, Meissner I, Piepgras DG, Huston J, et al: Aspirin as a promising agent for decreasing incidence of cerebral aneurysm rupture. Stroke 42:3156-3162, 2011

27. Hovsepian DM, Ziporin SJ, Sakurai MK, Lee JK, Curci JA, Thompson RW: Elevated plasma levels of matrix metalloproteinase-9 in patients with abdominal aortic aneurysms: a circulating marker of degenerative aneurysm disease. J Vasc Interv Radiol 11:1345-1352, 2000

28. Hua Y, Xue J, Sun F, Zhu L, Xie M: Aspirin inhibits MMP-2 and MMP-9 expressions and activities through upregulation of PPARalpha/gamma and TIMP gene expressions in ox-LDL-stimulated macrophages derived from human monocytes. Pharmacology 83:18-25, 2009 
29. Huang J, van Gelder JM: The probability of sudden death from rupture of intracranial aneurysms: a meta-analysis. Neurosurgery 51:1101-1107, 2002

30. Hwang J, Saha A, Boo YC, Sorescu GP, McNally JS, Holland SM, et al: Oscillatory shear stress stimulates endothelial production of $\mathrm{O}_{2}{ }^{-}$from $\mathrm{p} 47^{\text {phox }}$-dependent NAD $(\mathrm{P}) \mathrm{H}$ oxidases, leading to monocyte adhesion. J Biol Chem 278:4729147298, 2003

31. Ishii $\mathrm{T}$, Asuwa $\mathrm{N}$ : Collagen and elastin degradation by matrix metalloproteinases and tissue inhibitors of matrix metalloproteinase in aortic dissection. Hum Pathol 31:640-646, 2000

32. Jamous MA, Nagahiro S, Kitazato KT, Tamura T, Aziz HA, Shono M, et al: Endothelial injury and inflammatory response induced by hemodynamic changes preceding intracranial aneurysm formation: experimental study in rats. $\mathbf{J}$ Neurosurg 107:405-411, 2007

33. Jayaraman T, Berenstein V, Li X, Mayer J, Silane M, Shin YS, et al: Tumor necrosis factor alpha is a key modulator of inflammation in cerebral aneurysms. Neurosurgery 57:558564,2005

34. Jin D, Sheng J, Yang X, Gao B: Matrix metalloproteinases and tissue inhibitors of metalloproteinases expression in human cerebral ruptured and unruptured aneurysm. Surg Neurol 68 (Suppl 2):S11-S16, 2007

35. Juvela S, Poussa K, Porras M: Factors affecting formation and growth of intracranial aneurysms: a long-term follow-up study. Stroke 32:485-491, 2001

36. Kanematsu Y, Kanematsu M, Kurihara C, Tada Y, Tsou TL, van Rooijen N, et al: Critical roles of macrophages in the formation of intracranial aneurysm. Stroke 42:173-178, 2011

37. Kataoka K, Taneda M, Asai T, Kinoshita A, Ito M, Kuroda R: Structural fragility and inflammatory response of ruptured cerebral aneurysms. A comparative study between ruptured and unruptured cerebral aneurysms. Stroke 30:1396-1401, 1999

38. Katsuda S, Okada Y, Okada Y, Imai K, Nakanishi I: Matrix metalloproteinase-9 (92-kd gelatinase/type IV collagenase equals gelatinase B) can degrade arterial elastin. Am J Pathol 145:1208-1218, 1994

39. Kim SC, Singh M, Huang J, Prestigiacomo CJ, Winfree CJ, Solomon RA, et al: Matrix metalloproteinase-9 in cerebral aneurysms. Neurosurgery 41:642-647, 1997

40. Kim ST, Brinjikji W, Lanzino G, Kallmes DF: Neurovascular manifestations of connective-tissue diseases: a review. Interv Neuroradiol 22:624-637, 2016

41. Kondo S, Hashimoto N, Kikuchi H, Hazama F, Nagata I, Kataoka H: Apoptosis of medial smooth muscle cells in the development of saccular cerebral aneurysms in rats. Stroke 29:181-189, 1998

42. Kosierkiewicz TA, Factor SM, Dickson DW: Immunocytochemical studies of atherosclerotic lesions of cerebral berry aneurysms. J Neuropathol Exp Neurol 53:399-406, 1994

43. Laaksamo E, Tulamo R, Liiman A, Baumann M, Friedlander $\mathrm{RM}$, Hernesniemi $\mathrm{J}$, et al: Oxidative stress is associated with cell death, wall degradation, and increased risk of rupture of the intracranial aneurysm wall. Neurosurgery 72:109-117, 2013

44. Lindholt JS, Vammen S, Fasting H, Henneberg EW, Heickendorff L: The plasma level of matrix metalloproteinase 9 may predict the natural history of small abdominal aortic aneurysms. A preliminary study. Eur J Vasc Endovasc Surg 20:281-285, 2000

45. Longo GM, Xiong W, Greiner TC, Zhao Y, Fiotti N, Baxter BT: Matrix metalloproteinases 2 and 9 work in concert to produce aortic aneurysms. J Clin Invest 110:625-632, 2002

46. McCarthy SM, Bove PF, Matthews DE, Akaike T, van der Vliet A: Nitric oxide regulation of MMP-9 activation and its relationship to modifications of the cysteine switch. Biochemistry 47:5832-5840, 2008

47. McMillan WD, Pearce WH: Increased plasma levels of metalloproteinase-9 are associated with abdominal aortic aneurysms. J Vasc Surg 29:122-127-129, 1999

48. Meng H, Tutino VM, Xiang J, Siddiqui A: High WSS or low WSS? Complex interactions of hemodynamics with intracranial aneurysm initiation, growth, and rupture: toward a unifying hypothesis. AJNR Am J Neuroradiol 35:1254-1262, 2014

49. Moriwaki T, Takagi Y, Sadamasa N, Aoki T, Nozaki K, Hashimoto N: Impaired progression of cerebral aneurysms in interleukin-1 $\beta$-deficient mice. Stroke 37:900-905, 2006

50. Pearce WH, Shively VP: Abdominal aortic aneurysm as a complex multifactorial disease: interactions of polymorphisms of inflammatory genes, features of autoimmunity, and current status of MMPs. Ann N Y Acad Sci 1085:117-132, 2006

51. Peters DG, Kassam A, St Jean PL, Yonas H, Ferrell RE: Functional polymorphism in the matrix metalloproteinase-9 promoter as a potential risk factor for intracranial aneurysm. Stroke 30:2612-2616, 1999

52. Raps EC, Rogers JD, Galetta SL, Solomon RA, Lennihan L, Klebanoff LM, et al: The clinical spectrum of unruptured intracranial aneurysms. Arch Neurol 50:265-268, 1993

53. Rinkel GJ, Djibuti M, Algra A, van Gijn J: Prevalence and risk of rupture of intracranial aneurysms: a systematic review. Stroke 29:251-256, 1998

54. Rojas HA, Fernandes KSDS, Ottone MR, Magalhães KCSF, Albuquerque LAF, Pereira JLB, et al: Levels of MMP-9 in patients with intracranial aneurysm: relation with risk factors, size and clinical presentation. Clin Biochem 55:63-68, 2018

55. Sakaki T, Kohmura E, Kishiguchi T, Yuguchi T, Yamashita T, Hayakawa T: Loss and apoptosis of smooth muscle cells in intracranial aneurysms. Studies with in situ DNA end labeling and antibody against single-stranded DNA. Acta Neurochir (Wien) 139:469-475, 1997

56. Schievink WI: Intracranial aneurysms. N Engl J Med 336:28-40, 1997

57. Schievink WI, Wijdicks EF, Parisi JE, Piepgras DG, Whisnant JP: Sudden death from aneurysmal subarachnoid hemorrhage. Neurology 45:871-874, 1995

58. Seo KW, Lee SJ, Ye BH, Kim YW, Bae SS, Kim CD: Mechanical stretch enhances the expression and activity of osteopontin and MMP-2 via the Akt1/AP-1 pathways in VSMC. J Mol Cell Cardiol 85:13-24, 2015

59. Shojima M, Oshima M, Takagi K, Torii R, Hayakawa M, Katada K, et al: Magnitude and role of wall shear stress on cerebral aneurysm: computational fluid dynamic study of 20 middle cerebral artery aneurysms. Stroke 35:2500-2505, 2004

60. Springman EB, Angleton EL, Birkedal-Hansen H, Van Wart HE: Multiple modes of activation of latent human fibroblast collagenase: evidence for the role of a Cys 73 active-site zinc complex in latency and a "cysteine switch" mechanism for activation. Proc Natl Acad Sci U S A 87:364-368, 1990

61. Starke RM, Chalouhi N, Ali MS, Jabbour PM, Tjoumakaris SI, Gonzalez LF, et al: The role of oxidative stress in cerebral aneurysm formation and rupture. Curr Neurovasc Res 10:247-255, 2013

62. Tallant C, Marrero A, Gomis-Rüth FX: Matrix metalloproteinases: fold and function of their catalytic domains. Biochim Biophys Acta 1803:20-28, 2010

63. Visse R, Nagase H: Matrix metalloproteinases and tissue inhibitors of metalloproteinases: structure, function, and biochemistry. Circ Res 92:827-839, 2003

64. Wilson WR, Anderton M, Choke EC, Dawson J, Loftus IM, Thompson MM: Elevated plasma MMP1 and MMP9 are 
associated with abdominal aortic aneurysm rupture. Eur J Vasc Endovasc Surg 35:580-584, 2008

65. Wilson WR, Anderton M, Schwalbe EC, Jones JL, Furness PN, Bell PR, et al: Matrix metalloproteinase- 8 and -9 are increased at the site of abdominal aortic aneurysm rupture. Circulation 113:438-445, 2006

\section{Disclosures}

Dr. Grandhi is a consultant for Medtronic Neurovascular, Cerenovus, and BALT Neurovascular. Dr. Taussky is a consultant for Medtronic, Stryker Neurovascular, and Cerenovus. Dr. Ducruet is a consultant for Medtronic, Cerenovus, and Penumbra.

\section{Author Contributions}

Conception and design: all authors. Drafting the article: all authors. Critically revising the article: all authors. Reviewed submitted version of manuscript: all authors. Approved the final version of the manuscript on behalf of all authors: Grandhi.

\section{Correspondence}

Ramesh Grandhi: Clinical Neurosciences Center, University of Utah, Salt Lake City, UT. neuropub@hsc.utah.edu. 